\title{
TRATAMIENTO DEL IGV AL COMERCIO EXTERIOR DE SERVICIOS EN EL PERÚ
}

\section{VAT treatment on foreign trade of services in Peru}

Óscar Vásquez Nieva*

Universidad San Ignacio de Loyola

Abogado por la Pontificia Universidad Católica del Perú. Magister en Comercio Exterior y Aduanas por la Universidad de Canberra (Australia). Docente de la Universidad San Ignacio de Loyola. Ex Director Ejecutivo de la Revista Derecho y Sociedad. Consultor en Comercio Exterior y Aduanas. ORCID iD: 0000-0001-5448-5785. Contacto: o_vasquez@outlook.com 


\section{Resumen:}

El IGV como impuesto que incide en el tráfico comercial de bienes y servicios se circunscribe a una determinada territorialidad donde debe ocurrir el hecho imponible. En el Perú, la Ley del IGV establece que la Exportación de Servicios no está afecta a dicho impuesto, pero bajo determinadas condiciones donde resalta que el consumo del servicio por el sujeto no residente tiene que producirse íntegramente en el extranjero, a excepción de algunos supuestos señalados taxativamente en dicha Ley. Asimismo, conforme existe la Exportación de Servicios también podemos hablar coloquialmente de la Importación de Servicios o Utilización de Servicios prestados por sujetos no domiciliados, cuyo consumo o empleo se produce en territorio nacional independientemente del lugar de prestación.

\section{Abstract:}

The VAT as a tax that affects the trade traffic of goods and services is limited to a certain territory where the taxable event must occur. In Peru, the VAT Law establishes that the Exportation of Services is not affected by this tax, but under certain conditions where the one that highlights are that the consumption of the service by the non-resident person must have place entirely abroad. As there is Export of Services, we can also talk about Import of Services or Use of Services provided by a non-resident person, but whose consumption or use occurs in our domestic territory regardless of the place of provision.

Palabras clave:

IGV - Exportación de Servicios - Servicios - Comercio Exterior

Keywords:

VAT - Exportation of Services - Services - Foreign Trade

\section{Sumario:}

1. Antecedentes - 2. Los servicios - 3. La Ley N²9646 - 4. Nuestros comentarios a la legislación actual - 5 . Utilización (importación) de servicios -6 . Lista de referencias 


\section{ANTECEDENTES}

El 26 de Julio de 2017, el Congreso de la República aprobó la Ley N³0641¹, Ley que fomenta la Exportación de Servicios y el Turismo, la cual puso en vigencia el actual tratamiento tributario del Impuesto General a las Ventas (en adelante, IGV) a las Exportaciones de Servicios. $N^{\circ}$ 4682/2014-CR, mediante el cual se dispone reestablecer la vigencia de varios artículos de la Ley de Fomento al Comercio Exterior de Servicios que fueran derogados mediante los Decretos Legislativos $\mathrm{N}^{\circ} 1116$ y 1119. Sin embargo, como primer antecedente de ella tenemos a la Ley $N^{\circ}$ 29646, Ley de Fomento al Comercio Exterior de Servicios, aprobada también por el Congreso de la Republica, y puesta en vigencia el 1 de enero de 2011.

Bajo esta última ley, se intentó crear por primera vez un marco normativo integral para la exportación de servicios, declarándola, además, como una industria "de interés nacional, por ser un sector estratégico y prioritario del Estado peruano para la generación de empleo calificado, divisas e inversión en el país"2.

Asimismo, la Ley $N^{\circ} 29646$ precisó que los sectores competentes para crear las políticas para impulsar el comercio exterior de servicios son el Ministerio de Economía y Finanzas (en adelante, MEF) y el Ministerio de Comercio Exterior y Turismo (en adelante, MINCETUR).

En tal sentido, debemos señalar que, de acuerdo con la Ley N²7790, Ley de Organización y Funciones del MINCETUR, este ministerio se encarga de dirigir la política de comercio exterior y de turismo, asimismo, es responsable de promover las exportaciones y negociaciones comerciales, en coordinación con el MEF y la Cancillería, y los otros sectores del Poder Ejecutivo, cuando corresponda.

Por su parte, de acuerdo con el artículo 5 del Decreto Legislativo $N^{\circ} 183$, el MEF está encargado de planear, dirigir y controlar los asuntos relativos a la tributación y a la política arancelaria (en este último caso, en coordinación con el MINCETUR y los sectores involucrados, de ser el caso).

En este sentido, desde el año 2011 estos sectores han venido trabajando políticas sistémicas para la promoción de la exportación de servicios, apuntando a la eliminación de los sobrecostos tributarios para la prestación de servicios a

Vigente a partir del 1 de septiembre de 2017.

Artículo 1 de la Ley N²9646, Ley de Fomento al Comercio Exterior de Servicios. 
favor de residentes de otro Estado, la cual constituye una fuente de generación de divisas y de puestos de trabajo para profesionales y técnicos.

Estos sobrecostos (más del $18 \%$ del precio final del servicio, teniendo en cuenta también el crédito fiscal acumulado) frenan el crecimiento de los servicios que el Perú podría prestar al resto del mundo porque perdemos competitividad en relación a los prestadores del mismo servicio domiciliados en otros territorios.

Cabe mencionar que con la Ley N²9646 teníamos una legislación que consideraba como exportación de servicios exclusivamente a una cantidad limitada de actividades señaladas en el artículo 33 y en el derogado Apéndice $\checkmark$ de la Ley del IGV ${ }^{3}$ (en adelante, la Ley), principalmente referida al modo Suministro Transfronterizo de la OMC (cuando el servicio se brinda desde un país hacia el otro país, sin estar presente el Prestador).

En este sentido, nuestra legislación del IGV limitaba el desarrollo del sector servicios porque solamente unos cuantos servicios de los 155 tipos de servicios clasificados por la $\mathrm{OMC}^{4}$ tenían el potencial de ser considerados como exportación.

\section{LOS SERVICIOS}

El comercio internacional y, en particular las operaciones de exportación, no han sido un tema ajeno al legislador nacional. En nuestro país existe una variada normatividad que busca consagrar una política de promoción de la exportación, la cual responde a que ésta ofrece una serie de beneficios para el Estado, en tanto repercute directamente en la generación de empleo y riqueza.

Por tal razón, hoy en día tenemos en el Perú una regulación de exportaciones que apunta a: i) mejorar y hacer más competitiva la oferta exportable nacional y ii) superar problemas de posible doble tributación que conlleve la exportación de tributos (específicamente, en el caso de los impuestos al consumo).

Para ser exactos, la doble imposición también perjudica la competitividad de nuestra oferta exportable, con los consiguientes detrimentos en la economía del país, puesto que, si se grava la exportación de bienes y servicios de

Derogado mediante la Única Disposición Complementaria Derogatoria de la Ley №30641.

$4 \quad$ La Lista de Clasificación Sectorial de los Servicios se encuentra contenida en el documento MTN. GNS/W/120 de la OMC, del 10 de julio de 1991, la cual establece 155 subsectores de servicios divididos en 12 categorías sectoriales. 
origen peruano, el exportador no podría competir en el país de destino con la producción de ese país, dado que, al tributo nacional habría que sumar, en forma acumulativa, los impuestos propios del país de destino. De ser este el caso, si el exportador decidiese asumir el impuesto recortando su ganancia, el IGV se convertiría en un impuesto adicional y ciego a la renta con efectos confiscatorios $^{5}$.

En este sentido, debe indicarse que, en el Perú, el marco legal que rige el comercio exterior está conformado por nuestra Constitución Política del Perú, la cual proclama que el comercio exterior es libre; la Resolución Legislativa $N^{\circ} 26407$ mediante la cual aprobamos el acuerdo por el que se establece la Organización Mundial de Comercio (en adelante, OMC) y sus Acuerdos Comerciales Multilaterales; el Decreto Supremo N060-91-EF, el cual deroga el Registro Unificado de Exportación como requisito para hacer operaciones aduaneras; y el Decreto Legislativo $N^{\circ}$ 668, Ley Marco del Comercio Exterior, mediante el cual el Estado otorga garantías para el libre comercio exterior e interior, y a los agentes económicos, el libre acceso a la prestación de servicios, entre otras garantías.

En relación con los servicios, cabe precisar que si bien, técnicamente no se pueden exportar en consideración a su naturaleza incorpórea, por lo cual no pueden ser pasibles de un control aduanero aplicable a la carga, hablamos de exportación de servicios de manera coloquial. Aunque con más precisión podríamos referirnos a ellos como servicios transfronterizos, transnacionales o internacionales.

Sin perjuicio de ello, tal como se encuentra regulado el IGV en el caso de bienes, la inafectación para la exportación de servicios encuentra su fundamento en que el país de consumo de un servicio difiere del país de su producción o del domicilio del prestador; es decir, el consumo del servicio ocurre en el exterior y no en el país de origen del servicio.

Por su parte, los servicios presentan cuatro características que, en principio, los diferencian en gran medida de los bienes tangibles (Zeithaml et al, 2009, p. 21-22). Estas son:

i. Intangibilidad: falta de atributos físicos (no se pueden ver ni tocar).

ii. Inseparabilidad: simultaneidad en la entrega y el consumo del servicio.

Para mayor abundamiento, Sotelo (2017). Asimismo, el lector podría revisar las sentencias del Tribunal Constitucional recaídas sobre los Expedientes N²727-2002-AA/TC y N0004-2004-Al/ TC. 
iii. Perecibilidad: expresa que el servicio no puede ser almacenado.

iv. Heterogeneidad: refleja la variabilidad en la prestación del servicio, debido a que estos son provistos por personas, que nos desempeñamos inconsistentemente en el tiempo.

En este sentido, se considera comercio de servicios a un corte de cabello, un concierto de música, una cirugía estética, el transporte de una mercancía de un lugar a otro, la reparación y mantenimiento de equipos, publicidad, consultorías, entre otras actividades.

A nivel internacional, tenemos como marco al Acuerdo General sobre el Comercio de Servicios (en adelante, AGCS) de la OMC, vigente desde el 1 de enero de 1995, el cual se firma para crear un sistema multila teral aplicable al comercio internacional de servicios, como el Acuerdo General sobre Aranceles Aduaneros y Comercio lo hizo para el comercio de bienes. El Perú es miembro de la OMC y, por lo tanto, signatario del AGCS ${ }^{6}$.

La finalidad del Acuerdo General sobre el Comercio de Servicios es suprimir las barreras comerciales que afectan las cuatro modalidades de prestación de los servicios ${ }^{7}$ :

i. Suministro transfronterizo: cuando el servicio se brinda desde un país hacia el otro país, sin estar presente el prestador (por correo, internet, etc.).

ii. Consumo en el extranjero: cuando el consumidor se desplaza de su país a otro país para obtener el servicio, por ejemplo, un enfermo que viaja a otro país para que le presten un tratamiento médico.

iii. Presencia comercial: cuando un proveedor de servicios de un país se constituye físicamente en otro país, para prestar allí sus servicios.

iv. Presencia de personas físicas: de manera semejante a la presencia comercial, pero se aplica para las personas naturales en lugar de empresas, tales como profesionales o técnicos.

$6 \quad$ El Perú es signatario del GATT desde 1951 y es miembro fundador de la OMC desde 1995. Los acuerdos de la OMC fueron incorporados en la legislación nacional mediante Resolución Legislativa N² 26407 de 1994. Asimismo, actualmente el Perú y otras 22 economías, que incluyen a la Unión Europea y los Estados Unidos, se encuentran negociando un nuevo Acuerdo de Servicios (conocido como TISA por sus siglas en inglés, Trade in Servicies Agreement) con el fin de continuar con la liberación de barreras al comercio de servicios.

7 Numeral 2 del artículo I de la Parte I del AGCS. 
Cabe precisar que en las dos primeras modalidades el prestador de servicios no se desplaza físicamente al otro país, lo contrario sucede en las dos últimas modalidades, en las cuales el suministrador se moviliza a los países donde se reciben los servicios, bien mediante un establecimiento permanente o el desplazamiento temporal.

\section{LA LEY $\mathbf{N}^{\circ} 29646$}

Por las razones mencionadas, mediante la Ley N²29646, Ley de Fomento al Comercio Exterior de Servicios, el Congreso de la Republica decidió adecuar nuestra legislación tributaria del IGV a 3 de los 4 modos de comercio internacional de servicios que la OMC reconoce:

i. Comercio transfronterizo (actividades en el literal A del derogado Apéndice $\checkmark$ de la Ley del IGV): el cuál era el único modo que ya estaba contemplado con anterioridad. Su tratamiento: desgravación y devolución del crédito fiscal como saldo a favor del exportador.

ii. Consumo en el extranjero 1 (actividades en el literal B del derogado Apéndice $V$ de la Ley del IGV): cuando el consumidor extranjero (empresa) se desplaza y adquiere el servicio en el Perú, como, por ejemplo, en el caso de reparación de naves de bandera extranjera en nuestro puerto del Callao. Su tratamiento: desgravación y devolución del crédito fiscal como saldo a favor del exportador.

iii. Consumo en el extranjero 2 (actividades en el literal $C$ del derogado Apéndice $V$ de la Ley del IGV): cuando el consumidor extranjero (persona natural) se desplaza y adquiere el servicio en el Perú, como, por ejemplo, en el caso de servicios a turistas no domiciliados. Su tratamiento: se grava la operación con IGV, pero se devolvería el impuesto al momento del embarque del turista de regreso a su país.

iv. Presencia de personas físicas (Actividades en el Literal D del derogado Apéndice $V$ de la Ley del IGV): traslado de profesionales o técnicos del Perú a otro país para prestar sus servicios, como en el caso de docentes peruanos que viajan al exterior para el dictado de una conferencia. Su tratamiento: inafectación (por la extraterritorialidad de la operación), otorgándose la devolución del crédito fiscal acumulado vía saldo a favor del exportador.

v. Aquellas actividades señaladas en el artículo 33 de la Ley, las cuales, por mandato de la ley (legal), se consideran exportación de servicios (o exportaciones fictas) permanecieron vigentes. 
El cuarto modo OMC, presencia comercial (en este caso, el sujeto domiciliado crea en otro país una sucursal o establecimiento permanente para prestar sus servicios), no fue regulado dado que estas actividades se producen fuera del territorio nacional, por lo cual el IGV no les alcanza.

Sin embargo, mediante publicación efectuada en el Diario Oficial El Peruano, el 7 de julio de 2012, fue derogada la mayor parte de la Ley N² 29646 por la Única Disposición Complementaria Derogatoria del Decreto Legislativo $N^{\circ}$ 1116, cuando aún no había entrado en vigencia la mayoría de sus disposiciones que estaban sujetas a reglamentación.

\section{NUESTROS COMENTARIOS A LA LEGISLACIÓN ACTUAL}

Tradicionalmente, tratándose de aquellos tributos que tienen por objeto el tráfico de bienes (tributos de naturaleza real), el elemento de conexión es el lugar donde se realiza el hecho imponible (principio de territorialidad), a diferencia de los tributos que inciden sobre la obtención de renta (tributos de naturaleza personal), en donde el criterio de sujeción no es de tipo objetivo sino subjetivo (residencia o domicilio, por ejemplo).

Así tenemos que el principio de territorialidad queda consagrado en nuestra legislación del IGV en virtud de lo dispuesto en el artículo 1 de la Ley ${ }^{8}$ que, al regular el ámbito de aplicación del impuesto, señala que constituyen operaciones gravadas con dicho impuesto, entre otros, la venta de bienes muebles en el país (inciso a), la prestación de servicios en el país (inciso b) y la importación de bienes (inciso e). En tal sentido, nuestro IGV es estructuralmente un impuesto del tipo valor agregado que tiene por objeto el tráfico mercantil de bienes y servicios ${ }^{9}$ dentro de nuestro territorio nacional.

Sin perjuicio de ello, a fin de establecer los contornos precisos de la territorialidad que ha asumido nuestra legislación del IGV, la lectura del mencionado artículo 1 debe complementarse con la del artículo 33 de la misma Ley, la cual establece que ni la exportación de bienes ni la de servicios, entre otros supuestos, están afectos al IGV.

$8 \quad$ Nos referiremos a la "Ley" o "Ley del IGV" para señalar al Texto Único Ordenado (TUO) de la Ley del Impuesto General a las Ventas e Impuesto Selectivo Al Consumo, aprobado mediante Decreto Supremo $N^{\circ}$ 055-99-EF, incluyendo todas las modificaciones que hayan afectado a esta Ley y a su TUO.

9 Para mayor abundamiento, recomendamos la lectura del capítulo "El Hecho Imponible en el IGV", Villanueva (2014). 
En tal sentido, del análisis conjunto de tales artículos fluye que nuestro IGV ha optado por la aplicación del denominado principio de imposición en el País de Destino.

Al respecto, debe precisarse que, en materia de imposición indirecta sobre el comercio internacional, a fin de evitar la ocurrencia de una doble imposición, se han creado dos variantes a fin de determinar cuál debe ser el Estado que debe percibir el gravamen al consumo proveniente de una transacción interjurisdiccional ${ }^{10}$.

La primera de ellas, denominada principio de imposición en el país de origen, considera que sólo debe aplicarse el gravamen al consumo en el país en el que se hubiera elaborado o prestado el bien o el servicio, respectivamente. Como consecuencia de ello, se grava la exportación con la imposición al consumo y, correlativamente, se desgrava la importación de bienes o servicios al país.

Por su parte, en virtud del principio de imposición en el país de destino, el Estado en donde se grava una operación transfronteriza es aquél en donde ocurre el consumo del bien o servicio, por lo que la exportación del bien o servicio realizada en el país de su producción no se grava con el impuesto al consumo, afectándose, en contrario, la importación de bienes y servicios.

En este sentido, tratándose de la imposición sobre el valor agregado, como es el caso de nuestro IGV, podemos ver en la legislación comparada que los países optan mayoritariamente por la aplicación del principio de imposición en el país de destino, ya que se le considera el más idóneo para el adecuado funcionamiento del comercio exterior (Villanueva, 2014, p.477), por cuanto contribuye con la neutralidad fiscal en las transacciones de comercio internacional, en la medida que elimina las diferencias o distorsiones que genera la variedad de sistemas de imposición de cada país, procura colocar en igualdad de tratamiento a los bienes y servicios nacionales y a los importados, e incentiva los procesos de integración supranacional. Asimismo, en el caso de los servicios se apunta a ganar en competitividad.

10 "Respecto a la vinculación entre el sujeto activo y el sujeto pasivo, en materia de comercio internacional o interjurisdiccional, rigen dos criterios en cuanto a qué Estado debe percibir los impuestos al consumo: el de destino y el de origen. De acuerdo con el primero de estos criterios, los productos deben salir libres de impuestos del lugar en donde se fabricaron, debiendo gravarlo el país de destino, donde se ha de consumir el bien. Según el segundo de estos criterios, el de origen, los impuestos deben ser pagados al país o jurisdicción donde se fabricó el bien, eximiéndoselos en el lugar donde se consumen, o sea en el de destino" (Fenochietto, 2017, pp. 1147-1148). 
Por ello, conforme hemos indicado, el primer párrafo del artículo 33 de la Ley del IGV establece que la exportación de servicios no está afecta a dicho impuesto, pero bajo determinadas condiciones, entre las que resalta la que nos indica que el consumo (entendido como el uso, la explotación o el aprovechamiento) de los servicios prestados por el no residente debe darse íntegramente fuera del territorio nacional, dejando de lado aquellos casos en los que el consumo se produce parcialmente en el territorio nacional, como veremos más adelante.

En relación con ello, cabe mencionar que, hasta antes de la entrada en vigencia de la Ley N³0641, el artículo 33 de nuestra Ley del IGV sólo otorgaba la condición de exportación de servicios a un listado positivo de actividades, contenidos expresamente en el apéndice $V$ de dicha norma. En efecto, sólo podían constituir exportación de servicios aquéllos descritos taxativamente en esta reducida lista de actividades, excluyéndose así una gran cantidad de servicios que en la actualidad se producen en la economía y que son susceptibles de ser consumidos en el exterior.

Es así que, a partir de la entrada en vigencia de la Ley Nº 30641 se supera dicha situación con la derogación del Apéndice $V$ de la Ley del IGV, disponiéndose que a partir de la fecha de su entrada en vigencia ${ }^{11}$, cualquier servicio que cumpla con los requisitos establecidos en el artículo 33 de la Ley, es susceptible de calificar como exportación de servicios y, por tanto, inafectarse del IGV y otorgar al exportador el reintegro del saldo a favor del IGV.

Por otro lado, cabe destacar que el Congreso de la Republica, al poner en vigencia la Ley $N^{\circ} 30641$, no siguió el modelo de la Ley $N^{\circ} 29646$ en lo referido al requisito del consumo dentro del territorio nacional.

En efecto, en esta nueva modificación de la Ley, se mantuvo la aplicación del principio de imposición en el país de destino entendido de manera estricta, esto es que el uso, la explotación y/o el aprovechamiento de los servicios prestados por el no residente deben darse exclusivamente en el extranjero, dejando de lado a aquellos casos en que el consumo se produce parcialmente en el territorio nacional, aun cuando su vocación sea darse casi íntegramente en el extranjero, como, por ejemplo, en el caso de los servicios médicos o de salud, restándole competitividad a esta industria.

11 Fecha de entrada en vigencia: 1 de setiembre de 2017. 
En tal sentido, mediante la Ley $N^{\circ} 30641$, se modifica el quinto párrafo del artículo 33 de la Ley para señalar que los servicios se considerarán exportados en tanto cumplan concurrentemente con los siguientes requisitos:

- Que se presten a título oneroso.

- Que el exportador sea un sujeto domiciliado en el país.

- Que el usuario sea un sujeto no domiciliado en el país.

- Que el consumo (entendido como el uso, la explotación y/o el aprovechamiento) de los servicios por parte del no residente debe darse íntegramente fuera del territorio nacional.

Asimismo, la norma agregó que el usuario tiene que estar, previamente, inscrito en el Registro de Exportadores de Servicios de la SUNAT.

Otras de las innovaciones que trajo consigo la Ley $N^{\circ} 30641$ fue que introdujo una definición legal sobre cuándo debería entenderse que el "uso, explotación o aprovechamiento" de los servicios ocurren en el exterior, ya que ni la Ley del IGV ni su reglamento definían de manera expresa qué debería entenderse al respecto.

En tal sentido, en virtud de la Segunda Disposición Complementaria Final de la Ley $N^{\circ} 30641$, se superó esta insuficiencia en la normatividad del IGV, disponiéndose expresamente lo siguiente:

Para efecto de lo dispuesto en el literal d) del quinto párrafo del Artículo 33 del Texto Único Ordenado de la Ley del Impuesto General a las Ventas e Impuesto Selectivo al Consumo, aprobado mediante el Decreto Supremo 055-99-EF y normas modificatorias, para establecer que el uso, explotación o aprovechamiento de los servicios por parte del no domiciliado tiene lugar en el extranjero se evaluarán las condiciones contractuales de cada caso en particular, a fin de determinar qué han estipulado respecto del lugar donde se lleva a cabo el primer acto de disposición del servicio, entendido como el beneficio económico inmediato que este genera al usuario no domiciliado.

Sin perjuicio de ello, cabe mencionar que esta falta de precisión de la legislación del IGV había sido cubierta por el Tribunal Fiscal mediante la Resolución del Tribunal Fiscal (RTF, en adelante) N²25-5-2000, la cual señalaba que: "el modo de establecer si un servicio es utilizado el país, es decir, si es consumido o empleado en el territorio nacional, es en función del lugar donde el usuario -el que contrató el servicio- llevará a cabo el primer acto de disposición del mismo". 
En este sentido, si bien esta resolución estaba relacionada a un caso de utilización de servicios, era opinión generalizada, compartida con la SUNAT mediante el Informe N²28-2004-SUNAT/2B0000, que también debería usarse el mismo criterio para determinar cuándo se configura la operación de exportación de servicios.

Por su parte, mediante el Decreto Supremo N³42-2017-EF se modificó el numeral 1 del artículo 9 del reglamento de la Ley, para señalar que para efectos de dar cumplimiento a los requisitos que deben observar los servicios para considerarse exportados, se deberá cumplir con que:

i. La prestación debe darse a título oneroso, y el pago debe considerarse renta de tercera categoría según la Ley del Impuesto a la Renta, aún si no se encuentra afecta a este impuesto.

ii. A efectos de ser considerados prestados desde el país hacia el exterior, los servicios deberán cumplir conjuntamente con lo siguiente:

a) La prestación debe realizarse íntegramente en el Perú.

b) El uso, explotación o aprovechamiento del servicio debe darse fuera del territorio nacional (para lo cual se considerará el lugar donde sucede el primer acto de disposición, es decir, el beneficio económico inmediato que genera al usuario no residente), evaluándose las condiciones contractuales y otros medios probatorios de cada caso en particular.

iii. El exportador de servicios debe, de manera previa a sus operaciones de exportación de servicios, estar inscrito en el registro de exportadores de servicios a cargo de la SUNAT.

\subsection{Las exportaciones de servicios fictas}

Adicionalmente a los servicios que por su propia naturaleza pueden cumplir con los requisitos señalados en el quinto párrafo del artículo 33 de la Ley del IGV, tenemos que el mencionado artículo 33 también le otorga expresamente la calidad de exportación a una serie de actividades que, a pesar de no cumplir en estricto con estos requisitos, el legislador ha querido considerarlos también como un supuesto de exportación para efectos del IGV.

En tal sentido, el artículo 33 de la Ley del IGV señala que también se considerarán exportaciones a las siguientes actividades (de servicios):

i. Prestación de servicios de hospedaje y alimentación: se considera exportación la prestación de servicios de hospedaje y alimentación, a personas 
no residentes, en forma individual o en paquete turístico, durante su permanencia, por un plazo que no puede exceder de 60 días por cada ingreso al Perú, previa presentación de su Tarjeta Andina de Migración, y su pasaporte, salvoconducto o Documento Nacional de Identidad que, conforme con los tratados internacionales, sirvan para el ingreso al Perú.

ii. Transporte de pasajeros o mercancías del país hacia el exterior: se consideran exportaciones los servicios de transporte de pasajeros o bienes que los navieros o empresas navieras peruanos realicen desde el Perú hacia el exterior, y también los servicios de transporte de carga aérea que se realicen desde el Perú hacia el exterior.

iii. Mantenimiento de naves y aeronaves: se consideran exportaciones los servicios de transformación, reparación, mantenimiento y conservación de naves y aeronaves de bandera extranjera a favor de sujetos no residentes, siempre que su utilización económica se realice fuera del territorio nacional.

iv. Organización de paquete turístico: Se consideran exportaciones los servicios de alimentación (incluye servicios de alimentación tipo catering), traslados, transporte turístico (terrestre, aéreo, ferroviario, acuático, teleféricos y funiculares de pasajeros con origen y destino desde el mismo punto de embarque), espectáculos de folclore nacional, teatro, conciertos de música clásica, ópera, opereta, ballet, zarzuela, espectáculos deportivos declarados de interés nacional; museos, eventos gastronómicos, mediación u organización de servicios turísticos, servicios de traducción, turismo de aventura y otras actividades de turismo no convencional (turismo vivencial, social y rural comunitario) que conforman el paquete turístico prestado por operadores turísticos domiciliados en el país, a favor de agencias, operadores turísticos o personas naturales, no domiciliados en el país, en todos los $\operatorname{casos}^{12}$.

Asimismo, a propuesta del MINCETUR, por Decreto Supremo se podrán incorporar otros servicios para que puedan conformar el paquete turístico.

v. Servicios complementarios: se consideran exportaciones los servicios complementarios al transporte de carga realizada desde el Perú hacia el exterior y el que se realiza desde el exterior hacia el Perú, que sean necesarios para llevar a cabo dicho transporte, y siempre y cuando que se

12 De acuerdo con lo señalado en el numeral 9 de la Ley de IGV. 
realicen en la zona primaria aduanera ${ }^{13}$, cuando se presten a trasportistas de carga internacional o a otros sujetos no residentes en el país que sean titulares de la carga, acorde con la documentación aduanera de transito internacional.

Estos servicios complementarios al transporte de carga pueden ser (taxativamente ${ }^{14}$ ):

- Remolque

- Amarre o desamarre de boyas

- Alquiler de amarraderos

- Uso de área de operaciones

- Movilización de carga entre bodegas de la nave

- Transbordo de carga

- Descarga o embarque de carga o de contenedores vacíos

- Manipuleo de carga

- Estiba y desestiba

- Tracción de carga desde y hacia áreas de almacenamiento

- Practicaje

- Apoyo a aeronaves en tierra (rampa)

- Navegación aérea en ruta

- Aterrizaje-despegue

- Estacionamiento de la aeronave

13 De acuerdo con el artículo 2 de la Ley General de Aduanas, aprobada mediante el Decreto Legislativo $\mathrm{N}^{\circ} 1053$, se considera Zona Primaria a la parte del territorio aduanero que comprende los puertos, aeropuertos, terminales terrestres, centros de atención en frontera para las operaciones de desembarque, embarque, movilización o despacho de las mercancías y las oficinas, locales o dependencias destinadas al servicio directo de una aduana. Adicionalmente, puede comprender recintos aduaneros, espacios acuáticos o terrestres, predios o caminos habilitados o autorizados para las operaciones arriba mencionadas, incluyéndose a los almacenes y depósitos de mercancía que cumplan con los requisitos establecidos en la normatividad vigente, y hayan sido autorizados por la Administración Aduanera.

14 De acuerdo con lo señalado en el numeral 10 de la Ley de IGV. 
vi. Suministro de energía eléctrica: se considera exportación el suministro de energía eléctrica a favor de los sujetos domiciliados en las Zonas Especiales de Desarrollo (ZED) $)^{15}$, también llamadas Zonas Francas.

vii. Servicios prestados parcialmente en el extranjero: se considera exportaciones la prestación de servicios que se realizan parcialmente en el extranjero por sujetos generadores de Rentas de Tercera Categoría, según la Ley del Impuesto a la Renta, a favor de una persona no residente en el país, siempre que el uso, la explotación y/o el aprovechamiento haya ocurrido fuera del territorio nacional. Cabe mencionar que, para este supuesto, también es requisito que el exportador de servicios se encuentre inscrito en el Registro de Exportadores de Servicio de SUNAT previamente.

Asimismo, cabe precisar que el numeral 11 del artículo 9 del Reglamento de la Ley ha establecido que para que esta actividad califique como exportación de servicios debe cumplirse con los siguientes requisitos:

i) El servicio debe ser prestado por un sujeto residente generador de rentas de tercera categoría, aun cuando el mismo no se encuentre afecto a este impuesto.

ii) El servicio se considerará prestado parcialmente fuera del territorio nacional cuando la prestación del servicio sea realizada parte en el Perú y parte en el exterior.

iii) El uso, explotación o aprovechamiento del servicio debe realizarse (totalmente) en el extranjero.

En relación con lo señalado, como hemos visto antes, el legislador no regresó al modelo de la Ley $N^{\circ} 29646$ en lo referido a aceptar como actividades de exportación de servicios a aquéllas, cuyo consumo se realiza parcialmente en territorio nacional y parcialmente en el extranjero. Sin embargo, en el caso de

15 De acuerdo con el artículo 3 del Reglamento de las Zonas Especiales de Desarrollo, aprobado mediante Decreto Supremo Nº05-2019-MINCETUR, las ZED constituyen áreas geográficas debidamente delimitadas, destinadas a promover la estabilidad de las inversiones, fomentar el empleo, contribuir al desarrollo socioeconómico sostenible y promover la competitividad e innovación en las regiones donde se ubican. También se les denomina Zonas Francas, las cuáles son definidas en el artículo 2 de la Ley General de Aduanas, aprobada mediante el Decreto Legislativo $\mathrm{N}^{\circ} 1053$, como parte del territorio nacional debidamente delimitada, en la que las mercancías en ella introducidas se consideran como si no estuviesen dentro del territorio aduanero, para la aplicación de los derechos arancelarios, impuestos a la importación para el consumo y recargos a que hubiere lugar. 
estos supuestos taxativamente señalados en los numerales 4, 6, 7, 9, 10, y 11 del artículo 33, el requisito referido al consumo en el exterior no se cumple para que estas actividades sean consideradas exportación de servicios, puesto que el uso, explotación o aprovechamiento se va a producir en el territorio nacional bajo el criterio del primer acto de disposición que ya hemos mencionado.

\section{UTILIZACIÓN (IMPORTACIÓN) DE SERVICIOS}

Conforme existe la exportación de servicios, también podemos hablar coloquialmente de la importación de servicios (o utilización de servicios), tal cual está definida en el inciso b) del artículo 1 de la Ley del IGV, el cual señala que, entre otras operaciones, se encuentra gravada con IGV la utilización de servicios en el país prestados por sujetos no domiciliados.

La afectación de estos servicios con el IGV encuentra fundamento, como hemos señalado anteriormente, en primer lugar, por la aplicación del criterio de imposición al consumo exclusivo en el país de destino. Así, como ya hemos visto, el IGV como impuesto que incide en el tráfico comercial de bienes y servicios se adscribe a una determinada territorialidad que delimita la realización del hecho imponible previsto en la Ley.

Asimismo, la imposición se fundamenta también en el respeto al principio de neutralidad, pues un Impuesto al Valor Agregado, como nuestro IGV, no puede otorgar tratamientos diferenciados entre los servicios de origen nacional y los de origen extranjero.

En tal sentido, los servicios provenientes del exterior que son utilizados en el país deben quedar gravados en las mismas condiciones en que están los servicios de origen nacional. Es decir, se grava con IGV los servicios prestados desde el extranjero que son utilizados en el país, para equipararlos en la carga impositiva con respecto de los servicios prestados en el país por sujetos domiciliados. Por tanto, los servicios prestados por nacionales o extranjeros, para ser consumidos o utilizados en el país, con independencia de su origen, deben llevar la misma carga impositiva al momento de su oferta ${ }^{16}$.

Sin embargo, sí existe una diferencia respecto de lo que ocurre en el caso de la importación de bienes, en donde la condición de contribuyente se aplica

16 Por lo tanto, el IGV aplicado a la utilización de servicios de no domiciliados nunca podría ser más alto que el que se aplica a la prestación de servicios por parte de domiciliados para funcionar como una suerte de arancel aduanero, porque no se puede discriminar entre la producción nacional y la extranjera, en aplicación del principio de neutralidad del IGV. 
sobre cualquier sujeto que realice el ingreso de los bienes al país, sin exigirse que el mismo sea empresario o que realice actividades empresariales con habitualidad, dado que en el caso de la utilización de servicios sí se exige dicha condición.

En vista de ello, sólo los sujetos empresariales o aquellos sujetos que sin ser formalmente empresarios actúen como tales, es decir que sean habituales, se encontrarán gravados con el IGV cuando utilicen los servicios gravados.

Entonces, si los proveedores nacionales que prestan servicios deben gravar con IGV a sus consumidores, con lo cual el precio relativo de sus servicios se ve incrementado por este impuesto, de la misma forma cuando la operación sea brindada por proveedores extranjeros deberá también incluir el IGV (aunque en este caso será el consumidor quién deberá pagar el IGV al Estado). De modo tal que, el costo teórico (ceteris paribus) para el cliente o usuario del servicio será el mismo si contrata a un proveedor nacional o un proveedor extranjero.

\subsection{Características}

Conforme a lo dispuesto en el artículo 1 y el último párrafo del numeral 1 del inciso c) del artículo 3 de la ley del IGV, se encuentra gravada con este impuesto, la utilización de servicios en el país, entendiéndose por tal al servicio prestado por un sujeto no domiciliado para ser consumido en el país, independientemente del lugar en que ocurra el pago o perciba la contraprestación, y del lugar de celebración del contrato.

Asimismo, nos indican las normas citadas que se debe entender por servicio a "toda prestación que una persona realiza para otra y por la cual percibe una retribución o ingreso que se considere Renta de Tercera Categoría para los efectos del Impuesto a la Renta, aun cuando no esté afecto a este último impuesto, incluidos el arrendamiento de bienes muebles e inmuebles y el arrendamiento financiero" ${ }^{17}$.

Por otra parte, en relación con el aspecto subjetivo, cabe mencionar que conforme con el inciso c) del numeral 9.1 del artículo 9 de la Ley del IGV, los sujetos que utilicen en el país servicios prestados por no domiciliados tienen la calidad de contribuyentes del impuesto, precisándose, cómo mencionáramos antes, tratándose de aquellos sujetos que utilicen los servicios de no domiciliados y que no desarrollan actividad empresarial serán considerados como sujetos del impuesto, sólo cuando califiquen como habituales.

17 Informe N³67-2003-SUNAT/2B0000, emitido por la Intendencia Nacional Jurídico de la SUNAT. 
Al respecto, cabe precisar que el numeral 1 del artículo 4 del Reglamento de la Ley del Impuesto General a las Ventas establece en su tercer párrafo que, en el caso de la prestación de servicios, se debe considerar habituales aquellos servicios onerosos que sean similares a los de carácter comercial.

En este sentido, en la utilización de servicios opera la inversión del sujeto pasivo por lo que, como sujeto del impuesto en calidad de contribuyente, no queda designado el sujeto no domiciliado que presta el servicio, sino aquel sujeto que utiliza (consume) en el país los indicados servicios.

Para estos efectos, debe entenderse por sujeto no domiciliado a aquellos que contrario sensu, no califican como sujetos domiciliados en el país, de acuerdo con lo dispuesto en el artículo 7 de la Ley del Impuesto a la Renta, el cual establece quiénes son los sujetos que tienen esta calificación ${ }^{18}$.

Asimismo, nos señala el cuarto párrafo del literal b) del numeral 1 del artículo 2 del reglamento de la Ley del IGV que, para efectos de la utilización de servicios en el país, "se considera que el establecimiento permanente domiciliado en el exterior de personas naturales o jurídicas domiciliadas en el país, es un sujeto no domiciliado"19.

Por su parte, el nacimiento de la obligación tributaria se encuentra regulado en el inciso d) del artículo 4 de la Ley, el cual señala que, en la utilización

18 Artículo 7. Se consideran domiciliadas en el país:

a) Las personas naturales de nacionalidad peruana que tengan domicilio en el país, de acuerdo con las normas de derecho común.

b) Las personas naturales extranjeras que hayan residido o permanecido en el país más de ciento ochenta y tres (183) días calendario durante un periodo cualquiera de doce (12) meses.

c) Las personas que desempeñan en el extranjero funciones de representación o cargos oficiales y que hayan sido designadas por el Sector Público Nacional.

d) Las personas jurídicas constituidas en el país.

e) Las sucursales, agencias u otros establecimientos permanentes en el Perú de empresas unipersonales, sociedades y entidades de cualquier naturaleza constituidas en el exterior, en cuyo caso la condición de domiciliada alcanza a la sucursal, agencia u otro establecimiento permanente, en cuanto a su renta de fuente peruana.

f) Las sucesiones, cuando el causante, a la fecha de su fallecimiento, tuviera la condición de domiciliado con arreglo a las disposiciones de esta Ley.

g) Los bancos multinacionales a que se refiere la Décimo Sétima Disposición Final y Complementaria de la Ley General del Sistema Financiero y del Sistema de Seguros y Orgánica de la Superintendencia de Banca y Seguros- Ley $N^{\circ} 26702$, respecto de las rentas generadas por sus operaciones en el mercado interno.

h) Las empresas unipersonales, sociedades de hecho y entidades a que se refieren el tercer $y$ cuarto párrafos del Artículo $14^{\circ}$ de la Ley, constituidas o establecidas en el país.

19 Cuarto párrafo del literal b) del numeral 1 del artículo 2 del Reglamento de la Ley del IGV. 
de servicios, la obligación tributaria nacerá en la fecha de anotación del comprobante de pago en el Registro de Compras o en la fecha que ocurra el pago de la retribución, lo que ocurra primero. Asimismo, se precisa en el inciso c) del numeral 1 del artículo 3 del reglamento de la Ley del IGV que la fecha en que debemos considerar percibido un ingreso o retribución es aquella del pago o puesta a disposición de la contraprestación pactada, o aquella en la que se haga efectivo un documento de crédito, lo que ocurra primero.

Sin perjuicio de lo señalado, el tema más controversial y que genera una gran casuística es la determinación de si el consumo del servicio ha ocurrido en el Perú. Al respecto como ya hemos visto, el inciso c) del artículo 3 de la Ley del IGV indica que se entiende que el servicio es utilizado en el territorio nacional cuando, siendo prestado por un no domiciliado, sea consumido o empleado (usado, explotado o aprovechado) en el país, con prescindencia del lugar en que ocurra el pago o la contraprestación se perciba, así como del lugar de celebración del contrato.

En ese sentido, a fin de poder determinar si estamos ante una operación de utilización de servicios gravada con el IGV, lo que importará verificar es si el servicio prestado por el sujeto no domiciliado es consumido (usado, explotado o aprovechado) en el país, lo cual no necesariamente va a coincidir con el lugar en el que se presta el servicio, como, por ejemplo, en el caso de una consultoría de diseño realizada en una oficina de arquitectos situada en Alemania para ser utilizada por sus clientes en el Perú con miras a la construcción de una planta que va a operar fabricando prendas de vestir en nuestro país, donde tenemos que es en Alemania donde se presta el servicio, pero la utilización ocurre en el territorio nacional. Por lo tanto, a diferencia de lo que sucede con la exportación de servicios en general, no resulta relevante que el servicio haya sido prestado parte en el país y parte en el exterior, o íntegramente fuera del país o íntegramente en el Perú.

En relación con ello, el Tribunal Fiscal, mediante la RTF Nº 225-5-2000 que ya hemos revisado anteriormente, señaló que el modo de determinar si el servicio es utilizado en el país; es decir, si es consumido o empleado (usado, explotado o aprovechado) en el país, es en función del lugar donde el usuario que contrató el servicio, llevará a cabo el primer acto de disposición del mismo.

En el mismo sentido, a través del ya mencionado Informe $N^{\circ} 228-2004-$ SUNAT/2B0000, la Administración Tributaria compartió el criterio del Tribunal Fiscal al señalar que "puede afirmarse que para determinar si un servicio es consumido o empleado en el territorio nacional, deben analizarse, en primer lugar, las condiciones contractuales de cada caso en particular y, en base a 
ello, establecer el lugar dónde se lleva a cabo el primer acto de disposición del servicio".

Asimismo, nos señala el tercer párrafo del literal b) del numeral 1 del artículo 2 del reglamento de la Ley del IGV que "no se considerarán utilizados en el país aquellos servicios de ejecución inmediata que por su naturaleza se consumen íntegramente en el exterior, ni los servicios de reparación y mantenimiento de naves y aeronaves y otros medios de transporte prestados en el exterior" 20 .

\section{LISTA DE REFERENCIAS}

Fenochietto, R. (2007). El Impuesto al Valor Agregado. Editorial La Ley.

Sotelo, E. (2017). Notas para una aproximación a la aplicación del principio de no confiscatoriedad en materia tributaria. Revista lus et Veritas, 35, pp. 182-216.

Villanueva, W. (2014). Manual del IGV, Régimen General y Especiales. Pacifico Editores.

Zeithaml, V., Bitner, M. y Gremler, D. (2009). Marketing de Servicios. McGraw-Hill.

20 Tercer párrafo del literal b) del numeral 1 del artículo 2 del Reglamento de la Ley del IGV. 